\title{
МУЛЬТИСИСТЕМНЫЙ ВОСПАЛИТЕЛЬНЫЙ СИНДРОМ, АССОЦИИРОВАННЫЙ С COVID-19 У ДЕТЕЙ, КАК ИСХОД ЛЕГКИХ ФОРМ ЗАБОЛЕВАНИЯ
}

Мелехина Е.В. ${ }^{1}$, Музыка А.Д. ${ }^{1}$, Николаева С.В. ${ }^{1}$, Усенко Д.В. ${ }^{1}$, Понежева Ж.Б. ${ }^{1}$, Мирзонов В.А. ${ }^{3}$, Барыкин В.И. ${ }^{3}$, Новиков Д.В. ${ }^{3}$, Горелов A.B. ${ }^{1,2}$

${ }^{1}$ ФБУН «Центральный НИИ эпидемиологии» Роспотребнадзора, Москва

2 ФГАОУ ВО Первый МГМУ им. И.М. Сеченова Минздрава России (Сеченовский Университет)

${ }^{3}$ ГАУЗ МО «Химкинская ОБ»

Резюме. Согласно данным литературы, новая коронавирусная инфекция COVID-19 у детей развивается реже (до 10\% в структуре инфицированных вирусом SARS-CoV-2) и протекает легче, чем у взрослых, а летальные исходы - единичны. Однако у детей, перенесших легкие формы COVID-19, может развиваться мультисистемный воспалительный синдром (MBC). Для МBC характерно поражение респираторного и желудочнокишечного тракта (боль в животе, рвота, диарея), менингеальные признаки и признаки поражения сердечно-сосудистой системы (неспецифические изменения на ЭКГ по типу миокардита, систолическая дисфункция в сочетании с низким систолическим артериальным давлением, но без развития тромбоза коронарных артерий), а также лейкопения с выраженной лимфопенией, тромбоцитопенией, повышением уровня ферритина и маркеров миокардита. Возможно появление кожной сыпи, хейлита, цервикальной аденопатии и менингизма. Развитие тяжелого МВС у детей может происходить через 2-6 недель после перенесенных легких форм COVID-19. Это диктует необходимость раннего назначения противовирусных средств для экстренной профилактики в очаге инфекции и лечения легких форм болезни у детей согласно действующим клиническим рекомендациям. 
Ключевые слова: мультисистемный воспалительный синдром, COVID-19, дети, лечение.

Согласно данным литературы, новая коронавирусная инфекция COVID-19 у детей развивается реже, чем у взрослых. В начале пандемии у детей относительно редко диагностировались симптомы COVID-19: первоначально считалось, что дети могут быть мало восприимчивы к этой болезни [1]. В Китае за период с 8 декабря по 20 февраля 2020 года были зарегистрированы случаи заболевания у $1 \%$ подростков и $1 \%$ детей в возрасте до 10 лет [2]. По мере прогрессирования пандемии COVID-19 были диагностировано всё больше случаев болезни у детей. Имеющиеся на сегодня данные свидетельствуют о том, что дети составляют до 10\% в структуре инфицированных вирусом SARS-CoV-2. В Российской Федерации (РФ) дети составляют 8,4\% зарегистрированных случаев COVID-19 [3].

COVID-19 у детей протекает легче, чем у взрослых, а летальные исходы - единичны. Данные, предоставленные 77 учреждениями здравоохранения из 21 европейской страны по результатам наблюдения 582 детей в возрасте от 3-х дней жизни до 18 лет и опубликованные в виде обзора в конце июня 2020 в журнале Lancet, показали, что дети и подростки, в целом, менее серьезно страдают от COVID-19, чем взрослые и, особенно, пожилые пациенты. Тяжелое течение COVID-19 редко встречается у маленьких детей, включая младенцев, несмотря на незрелость их иммунного ответа. Статистический анализ показал, что возраст моложе 1 месяца, мужской пол, признаки поражения нижних дыхательных путей в дебюте заболевания, наличие коморбидной патологии и сочетанная инфекция с другими респираторными вирусами достоверно повышают вероятность необходимости госпитализации в отделение реанимации и интенсивной терапии [4]. В РФ COVID-19 у детей протекает, в целом, благоприятно: легкая форма регистрируется у половины детей с COVID-19. Тяжелая форма болезни регистрируется крайне редко - в 0,2\% всех случаев, что существенно 
ниже, чем у взрослых (у 1\% заболевших в возрасте 18-40 лет, у 5\% в возрасте 41-64 года и у 1/5 людей старше 65 лет) [3].

Клинически COVID-19 у детей протекает с типичными симптомами острого респираторного заболевания: лихорадкой $(49,6 \%)$ и кашлем $(45,5-$ 50,8\%) [5]. До трети детей с клинически манифестными формами болезни могут иметь высокую температуру, но, в целом, ниже $39^{\circ} \mathrm{C}[3,6-8]$. В отличие от взрослых дети более склонны к появлению внереспираторных симптомов: чаще отмечаются диарея $(9,4 \%)$ и рвота $(7,3 \%)$ [9]. Отмечено, что в Европе проявления со стороны желудочно-кишечного тракта (ЖКТ) встречались до 25\% случаев, что значительно чаще по сравнению с описанными проявлениями COVID-19 в Китае (6\%) и США (9\%) [10]. Желудочно-кишечные симптомы обычно появлялись до развития типичных респираторных проявлений [11]. Вирус SARS-Cov-2 обнаруживался в биоптатах кишечника и стуле выздоровевших пациентов, что указывает на его тропизм к ЖКТ и объясняет внереспираторные симптомы, а также длительное вирусовыделение фекально-оральным путем [12].

У новорожденных детей COVID-19 чаще протекает тяжелее, чем у детей старшего возраста: доля пациентов с тяжелым течением составляет 12\% [13, 14]. При том, что вертикальная передача SARS-CoV-2 не доказана [14-17], у 84\% новорожденных с подтвержденным COVID-19 мать была инфицирована вирусом SARS-Cov-2 [18]. Существует гипотеза о том, что развитие и тяжесть инфекции у новорожденных связаны с возможной гипоксемией у инфицированной матери, что повышает риск асфиксии в родах и преждевременных родов $[12,14]$.

В настоящее время уточнение распространенности бессимптомного течения COVID-19 крайне актуально, так как бессимптомные носители являются источником передачи вируса SARS-CoV-2. Высказано предположение, что до 80\% инфицированных людей могут являться бессимптомными носителями инфекции [19]. Согласно отчету, основанному на данных о пассажирах на борту круизного лайнера Diamond Princess, доля 
бессимптомных форм составила 17,9\% [20]. В другом исследовании было показано, что 12,9\% (94/731) детей (70\% из которых были в возрасте от 6 до 15 лет), у которых из ротоглотки методом ПЦР была выделена PHK SARS$\mathrm{CoV}-2$, не имели симптомов болезни [21]. В РФ бессимптомное течение подтверждено у трети пациентов детского возраста [3].

Начиная с марта 2020 года начинают появляться сообщения о развитии у детей, перенесших легкие формы COVID-19, мультисистемного воспалительного синдрома (MBC) [22]. Группой ученых из Бергамо (Италия) на фоне пандемии COVID-19 зафиксировано ежемесячное увеличение регистрации Кавасаки-подобного заболевания в 30 раз по сравнению с предыдущими 5 годами [23]. К концу июня 2020 американские ученые сообщили о 186 подтвержденных случаях MBC, ассоциированного с COVID19, причем тяжелого течения болезни у этих детей не было [24]. В настоящее время показано, что МВС имеет ряд сходных черт с синдромом Кавасаки, описанным Томисаку Кавасаки в Японии в 1967 году, но также и целый ряд отличий. По сравнению с пациентами с классическими формами болезни Кавасаки дети с MBC на фоне COVID-19 были старше (средний возраст 10 лет), имели поражение респираторного тракта и ЖКТ (боль в животе, рвота, диарея), менингеальные признаки и признаки поражения сердечнососудистой системы (неспецифические изменения на ЭКГ по типу миокардита, систолическая дисфункция в сочетании с низким систолическим артериальным давлением, но без развития тромбоза коронарных артерий). Для них характерна лейкопения с выраженной лимфопенией, тромбоцитопенией и повышением уровня ферритина, а также маркеров миокардита. Из классических проявлений болезни Кавасаки фиксировали кожную сыпь, хейлит, цервикальную аденопатию и менингизм [25-28]. Также описан случай классического течения болезни Кавасаки у 6-месячного ребенка с COVID-19. Однако и в этом наблюдении эхокардиограмма была без каких-либо признаков коронарной дилатации или аневризмы, перикардиального выпота и нормальной функцией клапана и желудочка [27]. 
«Пусковым» механизмом $\mathrm{MBC}$ у детей в настоящий момент рассматривается COVID-19 и возникающее при этой болезни гипервоспаление. Одна из гипотез состоит в том, что эндотелиальный АСЕ2 может потенцировать воспалительный ответ при коронарных поражениях, вызывая эндотелиальную дисфункцию [28]. Механизмом повреждения миокарда является “цитокиновый шторм”, вызванный несбалансированным ответом провоспалительных и регуляторных Т-клеток [29, 30]. Ранее исследователями из Японии была показана связь другого коронавируса HCoV-229E с болезнью Кавасаки [31]. Таким образом, семейство коронавирусов, в том числе, особо вирулентный штамм SARS-CoV-2, может быть одним из пусковых механизмов МВС, связанного с индукцией мощного иммунного ответа организма хозяина и сопровождающегося системным воспалительным поражением мелких сосудов различных органов и систем у детей.

Заключение.

К осени 2020 года стало очевидно, что более 50\% (а среди детей эти цифры еще выше - до 80\%) случаев новой коронавирусной инфекции COVID-19 протекает в виде легких и бессимптомных форм. Однако развитие тяжелого МBC у детей через 2-6 недель после перенесенных легких форм COVID-19 диктует необходимость раннего назначения противовирусных средств для экстренной профилактики в очаге инфекции (для детей это, как правило, внутрисемейный очаг) и лечения легких форм болезни согласно действующим клиническим рекомендациям по профилактике и лечению коронавирусной инфекции, вызванной SARS-Cov2.

\section{Литература.}

1. Lee PI, Hu YL, Chen PY, Huang YC, Hsueh PR. Are children less susceptible to COVID-19? J Microbiol Immunol Infect 2020. Doi: 10.1016/j.jmii.2020.02.011. 
2. Dong Y, Mo X, Hu Y, Qi X, Jiang F, Jiang Z, et al. Epidemiological characteristics of 2143 pediatric patients with 2019 coronavirus disease in China. Pediatrics 2020. Doi: 10.1542/peds.2020-0702.

3. Горелов А.В., Николаева С.В., Акимкин В.Г. Коронавирусная инфекция COVID-19 у детей в Российской Федерации. Инфекционные болезни. 2020; 18(3): 15-20.

4. Florian Götzinger, Begoña Santiago-García, Antoni Noguera-Julián, Miguel Lanaspa, Laura Lancella, Francesca I Calò Carducci, Natalia Gabrovska, Svetlana Velizarova, Petra Prunk, Veronika Osterman, Uros Krivec, Andrea Lo Vecchio, Delane Shingadia, Antoni Soriano-Arandes, Susana Melendo, Marcello Lanari, Luca Pierantoni, Noémie Wagner, Arnaud G L’Huillier, Ulrich Heininger, Nicole Ritz, Srini Bandi, Nina Krajcar, Srđan Roglić, Mar Santos, Christelle Christiaens, Marine Creuven, Danilo Buonsenso, Steven B Welch, Matthias Bogyi, Folke Brinkmann, Marc Tebruegge. COVID-19 in children and adolescents in Europe: a multinational, multicentre cohort study Lancet Child Adolesc Health 2020 Published Online June 25, 2020 https://doi.org/10.1016/S2352-4642(20)30177-2.

5. Liguoro I, Pilotto $\mathrm{C}$, Bonanni $\mathrm{M}$, et al. SARS-COV-2 infection in children and newborns: a systematic review [published online ahead of print, 2020 May 18]. Eur J Pediatr. 2020;1-18. doi:10.1007/s00431-02003684-7.

6. Parri N, Lenge M, Buonsenso D, Coronavirus Infection in Pediatric Emergency Departments (CONFIDENCE) Research Group (2020) Children with Covid-19 in pediatric emergency departments in Italy. N Engl J Med. 10.1056/NEJMc2007617

7. Lu X, Zhang L, Du H, et al. SARS-CoV-2 infection in children. N Engl J Med. 2020;382:1663-1665. doi: 10.1056/NEJMc2005073.

8. Cai J, Xu J, Lin D, Yang, Xu L, Qu Z, Zhang Y, Zhang H, Jia R, Liu, Wang X, Ge Y, Xia A, Tian H, Chang H, Wang C, Li J, Wang J, Zeng M 
(2020) A case series of children with 2019 novel coronavirus infection: clinical and epidemiological features. Clin Infect Dis. 10.1093/cid/ciaa198.

9. Zhu H, Wang L, Fang C, Peng S, Zhang L, Chang G, Xia S, Zhou W. Clinical analysis of 10 neonates born to mothers with 2019-nCoV pneumonia. Transl Pediatr. 2020;9:51-60. doi: 10.21037/tp.2020.02.06.

10. Buonsenso D, Costa S, Sanguinetti M, Cattani P, Posteraro B, Marchetti S, Carducci B, Lanzone A, Tamburrini E, Vento G, Valentini P (2020) Neonatal late onset infection with severe acute respiratory syndrome Coronavirus 2. Am J Perinatol. 10.1055/s-0040-1710541.

11.Gu J, Han B, Wang J. COVID-19: gastrointestinal manifestations and potential fecal-oral transmission. Gastroenterology. 2020;158:15181519. doi: 10.1053/j.gastro.2020.02.054.

12.Maternal and neonatal outcomes of pregnant women with COVID-19 pneumonia: a case-control study | medRxiv. 10.1101/2020.03.10.20033605v1. Accessed 2 Apr 2020.

13.Zhu H, Wang L, Fang C, Peng S, Zhang L, Chang G, Xia S, Zhou W. Clinical analysis of 10 neonates born to mothers with 2019-nCoV pneumonia. Transl Pediatr. 2020;9:51-60. doi: 10.21037/tp.2020.02.06.

14. Lu Q, Shi Y. Coronavirus disease (COVID-19) and neonate: what neonatologist need to know. J Med Virol. 2020;92:564-567. doi: 10.1002/jmv.25740.

15.Buonsenso D, Costa S, Sanguinetti M, Cattani P, Posteraro B, Marchetti S, Carducci B, Lanzone A, Tamburrini E, Vento G, Valentini P (2020) Neonatal late onset infection with severe acute respiratory syndrome Coronavirus 2. Am J Perinatol. 10.1055/s-0040-1710541.

16.Lu Q, Shi Y. Coronavirus disease (COVID-19) and neonate: what neonatologist need to know. J Med Virol 2020. Doi: 10.1002/jmv.25740.

17. Francesco Licciardi, Giulia Pruccoli, Marco Denina, Emilia Parodi, Manuela Taglietto, Sergio Rosati, Davide Montin. SARS-CoV-2-Induced 
Kawasaki-Like Hyperinflammatory Syndrome: A Novel COVID Phenotype in Children. Pediatrics. 2020 May 21;e20201711. doi: 10.1542/peds.2020-1711.

18. Han MS, Seong M-W, Heo EY, Park JH, Kim N, Shin S, Cho SI, Park SS, Choi EH (2020) Sequential analysis of viral load in a neonate and her mother infected with SARS-CoV-2. Clin Infect Dis. 10.1093/cid/ciaa447.

19.Day M. Covid-19: four fifths of cases are asymptomatic, China figures indicate. BMJ 2020;369:m1375.

20.Mizumoto K, Kagaya K, Zarebski A, Chowell G. Estimating the asymptomatic proportion of coronavirus disease 2019 (COVID-19) cases on board the Diamond Princess cruise ship, Yokohama, Japan, 2020. Euro Surveill 2020;25:2000180.

21. Dong Y, Mo X, Hu Y, Qi X, Jiang F, Jiang Z, et al. Epidemiological characteristics of 2143 pediatric patients with 2019 coronavirus disease in China. Pediatrics 2020. Doi: 10.1542/peds.2020-0702.

22.Francesco Licciardi, Giulia Pruccoli, Marco Denina, Emilia Parodi, Manuela Taglietto, Sergio Rosati, Davide Montin. SARS-CoV-2-Induced Kawasaki-Like Hyperinflammatory Syndrome: A Novel COVID Phenotype in Children. Pediatrics. 2020 May 21;e20201711. doi: 10.1542/peds.2020-1711.

23. Lucio Verdoni, MD,a Angelo Mazza, MD, Annalisa Gervasoni, MD,a Laura Martelli, MD,a Maurizio Ruggeri, MD,a Matteo Ciuffreda, MD,b Ezio Bonanomi, MD,c and Lorenzo D'Antiga, MDa. An outbreak of severe Kawasaki-like disease at the Italian epicentre of the SARS-CoV-2 epidemic: an observational cohort study. Lancet. 2020 May 13doi: 10.1016/S0140-6736(20)31103-X.

24. L.R. Feldstein, E.B. Rose, S.M. Horwitz et at. Multisystem Inflammatory Syndrome in U.S. Children and Adolescents The New England Journal of Medicine Downloaded from nejm.org on July 7, 2020 June 29, 2020 DOI: 10.1056/NEJMoa2021680 
25. Zahra Belhadjer et at. Acute Heart Failure in Multisystem Inflammatory Syndrome in Children (MIS-C) in the Context of Global SARS-CoV-2 Pandemic. Circulation. 2020 May 17. doi: 10.1161/CIRCULATIONAHA.120.048360.

26.Elvia I Rivera-Figueroa, Roberto Santos, Scott Simpson, Padma Garg. Incomplete Kawasaki Disease in a Child With Covid-19. Indian Pediatr. 2020 May 9;S097475591600179.

27. Veena G Jones, Marcos Mills, Dominique Suarez, Catherine A Hogan, Debra Yeh, J Bradley Segal, Elizabeth L Nguyen, Gabrielle R Barsh, Shiraz Maskatia, Roshni Mathew. COVID-19 and Kawasaki Disease: Novel Virus and Novel Case. Hosp Pediatr. 2020 Jun;10(6):537-540. doi: 10.1542/hpeds.2020-0123.

28. Suowen Xu. COVID-19 and Kawasaki Disease in Children. Pharmacol Res. 2020 May 25: 104951. doi: 10.1016/j.phrs.2020.104951.

29.Puja Mehta, Daniel F McAuley, Michael Brown, Emilie Sanchez, Rachel S Tattersall, Jessica J Manson.

30.COVID-19: Consider Cytokine Storm Syndromes and Immunosuppression. Lancet. 2020 Mar 28;395(10229):1033-1034. doi: 10.1016/S0140-6736(20)30628-0.

31. Rohit S. Loomba, MD, Enrique Villarreal, MD, Saul Flores, MD. Covid-19 and Kawasaki syndrome: should we really be surprised? This is an Accepted Manuscript for Cardiology in the Young as part of the Cambridge Coronavirus Collection. Subject to change during the editing and production process. DOI: S1047951120001432

32. Kazuya Shirato, Yoshio Imada, Miyuki Kawase, Keiko Nakagaki, Shutoku Matsuyama, Fumihiro Taguchicorresponding. Possible involvement of infection with human coronavirus 229E, but not NL63, in Kawasaki disease. J Med Virol. 2014 Dec; 86(12): 2146-2153. Published online 2014 Apr 24. doi: 10.1002/jmv.23950. 